\title{
Microwave Soil Moisture Retrieval Under Trees
}

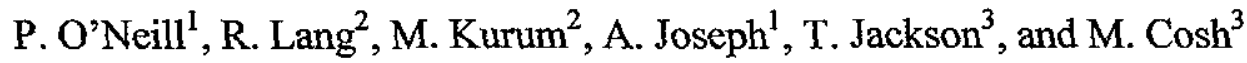 \\ ${ }^{1}$ Hydrological Sciences Branch / Code 614.3 \\ NASA Goddard Space Flight Center, Greenbelt, MD 20771 USA \\ tel: 1-301-614-5773, fax: 1-301-614-5808, Peggy.E.ONeill@nasa.gov \\ ${ }^{2}$ Dept. of Elect. \& Computer Engineering, George Washington University, Wash., DC 20052 USA \\ ${ }^{3}$ Hydrology \& Remote Sensing Laboratory, USDA ARS, Beltsville, MD 20705 USA
}

\begin{abstract}
Invited Paper to session \#I.13 on "Crop and Forest Monitoring with Microwave Radiometric Sensors"
\end{abstract}

ABSTRACT. Soil moisture is recognized as an important component of the water, energy, and carbon cycles at the interface between the Earth's surface and atmosphere. Current baseline soil moisture retrieval algorithms for microwave space missions have been developed and validated only over grasslands, agricultural crops, and generally light to moderate vegetation. Tree areas have commonly been excluded from operational soil moisture retrieval plans due to the large expected impact of trees on masking the microwave response to the underlying soil moisture. Our understanding of the microwave properties of trees of various sizes and their effect on soil moisture retrieval algorithms at $L$ band is presently limited, although research efforts are ongoing in Europe, the United States, and elsewhere to remedy this situation.

As part of this research, a coordinated sequence of field measurements involving the ComRAD (for Combined Radar/Radiometer) active/passive microwave truck instrument system has been undertaken. Jointly developed and operated by NASA Goddard Space Flight Center and George Washington University, ComRAD consists of dual-polarized $1.4 \mathrm{GHz}$ total-power radiometers (LH, LV) and a quad-polarized $1.25 \mathrm{GHz}$ L band radar sharing a single parabolic dish antenna with a novel broadband stacked patch dual-polarized feed, a quad-polarized $4.75 \mathrm{GHz} \mathrm{C}$ band radar, and a single channel $10 \mathrm{GHz}$ XHH radar. The instruments are deployed on a mobile truck with an 19-m hydraulic boom and share common control software; real-time calibrated signals, and the capability for automated data collection for unattended operation.

Most microwave soil moisture retrieval algorithms developed for use at $\mathrm{L}$ band frequencies are based on the tau-omega model, a simplified zero-order radiative transfer approach where scattering is largely ignored and vegetation canopies are generally treated as a bulk attenuating layer. In this approach, vegetation effects are parameterized by tau and omega, the microwave vegetation opacity and single scattering albedo. One goal of our current research is to determine whether the tau-omega model can work for tree canopies given the increased scatter from trees compared to grasses and crops, and if so, what are effective values for tau and omega for trees.

During 2006 and 2007 an experiment was conducted with a goal of optimizing microwave soil moisture retrieval algorithms for small to medium trees. Microwave measurements and supporting ground truth data (including size/angle distributions of tree constituents) were 
collected over stands of deciduous paulownia trees of different densities under full canopy and leaf-drop conditions. Using ComRAD measurements of the tree canopies and a vegetation scattering model [1], estimates of the tree layer attenuation and scattering albedo were obtained. In turn, these attenuation and scattering values were tested in the tau-omega model to retrieve soil moisture under the trees during three seasons (Spring to late Fall). The accuracy of microwave soil moisture retrieval under trees will be discussed, as well as the potential synergism between active and passive data at $\mathrm{L}$ band for improved soil moisture determination.

[1] N.S. Chauhan, D.M. Le Vine and R.H. Lang, "Discrete scatter model for microwave radar and radiometer response to corn: comparison of theory and data", IEEE Trans. Geosci. Remote Sensing, Vol. 32, No. 2, pp. 416-426, March 1994.

KEYWORDS: soil moisture, trees, retrieval algorithms, active/passive microwave 